\title{
Combining areal DXA bone mineral density and vertebrae postero-anterior width improves the prediction of vertebral strength
}

\author{
Grzegorz Tatoń • Eugeniusz Rokita • Andrzej Wróbel • \\ Mariusz Korkosz
}

Received: 4 March 2013 /Revised: 15 August 2013 /Accepted: 25 August 2013 /Published online: 1 October 2013

(C) The Author(s) 2013. This article is published with open access at Springerlink.com

\begin{abstract}
Objective Areal bone mineral density (aBMD) measured by dual-energy X-ray absorptiometry (DXA) is an important determinant of bone strength (BS), despite the fact that the correlation between aBMD and BS is relatively weak. Parameters that describe BS more accurately are desired. The aim of this study was to determine whether the geometrical corrections applied to aBMD would improve its ability for BS prediction. We considered new parameters, estimated from a single DXA measurement, as well as BMAD (bone mineral apparent density) reported in the literature.

Materials and methods In vitro studies were performed with the L3 vertebrae from 20 cadavers, which were studied with DXA and quantitative computed tomography (QCT). A mechanical strength assessment was carried out. Two new parameters were introduced: $v B M D_{\min }=\frac{a B M D}{W_{P A}^{m i n}}$ and $v B M D_{a v}=\frac{a B M D}{W_{P A}^{a v}}$ $\left(W_{P A}{ }^{\text {min }}\right.$-minimal vertebral body width in postero-anterior (PA) view, ${W_{P A}}^{a v}$ - average PA vertebral body width). Volumetric BMD measured by QCT (vBMD), aBMD, BMAD,
\end{abstract}

\footnotetext{
G. Tatoń $(\square) \cdot$ E. Rokita

Department of Biophysics, Jagiellonian University Medical College, Łazarza 16, 31530 Kraków, Poland

e-mail: g.taton@uj.edu.pl

E. Rokita

e-mail: ufrokita@cyf-kr.edu.pl
A. Wróbel
Institute of Physics, Jagiellonian University, Kraków, Poland
e-mail: awrobe@cyf-kr.edu.pl

\section{Korkosz}

Department of Internal Medicine and Gerontology,

Division of Rheumatology, Jagiellonian University Medical College,

Kraków, Poland

e-mail: mariusz.korkosz@uj.edu.pl
}

$\mathrm{vBMD}_{\text {min }}$, and $\mathrm{vBMD}_{\mathrm{av}}$ were correlated to ultimate load and ultimate stress $\left(\mathrm{P}_{\max }\right)$ to find the best predictor of vertebrae BS. Results The coefficients of correlation between $\mathrm{P}_{\max }$ and $\mathrm{vBMD}_{\min }, \mathrm{vBMD}_{\mathrm{av}}$, as well as BMAD, were $r=0.626$ $(p=0.005), r=0.610(p=0.006)$ and $r=0.567(p=0.012)$, respectively. Coefficients for vBMD and aBMD are $r=0.648$ $(p=0.003)$ and $r=0.511(p=0.03)$, respectively.

Conclusions Our results showed that aBMD normalized by vertebrae dimensions describes vertebrae BS better than aBMD alone. The considered indices $\mathrm{vBMD}_{\mathrm{av}}, \mathrm{vBMD}_{\text {min }}$, and BMAD can be measured in routine PA DXA and considerably improve $\mathrm{BS}$ variability prediction. $\mathrm{vBMD}_{\min }$ is superior compared to $\mathrm{vBMD}_{\mathrm{av}}$ and BMAD.

Keywords BMD · DXA · Fracture risk - QCT · Vertebrae strength

\section{Introduction}

Fracture risk (FR) assessment is essential in the evaluation of patients with low bone mass and is largely determined by bone strength (BS) [1]. Biomechanical bone properties depend upon bone composition, shape, size, and micro- and macroarchitecture [1-8]. Currently, only the femoral neck areal bone mineral density (aBMD) measured with dual-energy X-ray absorptiometry (DXA) is an optional component of the FRAX algorithm allowing for calculation of an individual 10-year FR [9]. aBMD is a surrogate marker of BS because the latter cannot be measured in vivo. The DXA technique does not measure BS directly but is simple, inexpensive, and easily accessible [3, 10]. A correlation between the FR and aBMD is unsatisfactory and accounts for only $60-70 \%$ of the variation of BS $[1,2,11,12]$, suggesting a need for other factors not captured in DXA, e.g., bone geometry. 
Considering clinical practice, the best BS predictor is volumetric bone mineral density (vBMD) $[6,8,13]$, which can be measured by quantitative computed tomography (QCT). Body or skeletal size affects the results of DXA because of its two-dimensional geometry. The QCT method does not have such limitations and is believed to be more reliable [10]. The QCT shows advantages compared to DXA, especially in vertebral studies; it is less affected by surrounding tissues, eliminates the posterior vertebrae elements, and facilitates analysis of trabecular and cortical bone separately [11].

Because of higher costs, lower accessibility, and large Xray exposure, QCT assessment probably would not displace the DXA technique, so efforts were made to improve DXA links with FR. The DXA technique can be applied for the assessment of vertebral vBMD after some geometric corrections. Such solutions were proposed in the 1990s [13, 14], but have also been discussed recently [10,15-18]. The idea relies on the use of vertebral body dimensions and aBMD measured in postero-anterior (PA) and/or supine lateral (LAT) views in order to estimate $\mathrm{vBMD}$.

One of the parameters described in the literature is bone mineral apparent density (BMAD) $[10,15,17,18]$. BMAD is the volumetric bone mineral density estimated from DXA and calculated as the ratio of aBMD and the square root of PA vertebrae area $(A): B M A D=a B M D / \sqrt{A}$. BMAD was developed as a potential resolution for a problem with the interpretation of DXA results in children and adolescents [10, $15,18]$ but was also used for aBMD adjustment in adults [17].

The aim of the present study was to check whether aBMD adjustment with the use of geometrical information improves its ability for BS variability prediction. The second aim was to propose a novel parameter calculated from a single and routine PA DXA measurement that would be a better predictor of vertebral BS and vertebral FR than aBMD. To the best of our knowledge, no works were performed to study the direct dependence between the mechanical BS and volumetric density estimated from DXA.

\section{Materials and methods}

All symbols used are presented in Table 1 and Fig. 1. Three is a simplified scheme of the vertebrae DXA measurement geometry presented in Fig. 1. In order to deliver appropriate formulas, the whole vertebral volume was divided into $\mathrm{N}$ small volume elements. The base area of each volume element is small and equal to a. The areal bone mineral density (aBMD) is defined as:

$a B M D=\frac{B M C}{A}=\frac{B M C}{a \cdot N}$
Table 1 The meaning of symbols used

\begin{tabular}{|c|c|c|}
\hline Symbol & Unit & Meaning \\
\hline $\mathrm{PA}$ & - & Posteroanterior \\
\hline LAT & - & Supine lateral \\
\hline FR & - & Fracture risk \\
\hline BS & - & Bone strength \\
\hline $\mathrm{BMC}$ & {$[\mathrm{g}]$} & Bone mineral content \\
\hline $\mathrm{P}_{\max }$ & {$[\mathrm{MPa}]$} & $\begin{array}{l}\text { The ultimate stress, the pressure causing the } \\
\text { vertebrae crush in the mechanical strength } \\
\text { tests }\end{array}$ \\
\hline $\mathrm{F}_{\max }$ & {$[\mathrm{kN}]$} & $\begin{array}{l}\text { The ultimate load, the force causing the vertebra } \\
\text { crush in the mechanical strength tests }\end{array}$ \\
\hline$A_{A}^{\min }$ & {$\left[\mathrm{cm}^{2}\right]$} & The minimal vertebrae axial cross-sectional area \\
\hline A & {$\left[\mathrm{cm}^{2}\right]$} & The total area of vertebral PA projection \\
\hline a & {$\left[\mathrm{cm}^{2}\right]$} & The base area of a single volume element \\
\hline $\mathrm{N}$ & - & Number of volume elements \\
\hline $\mathrm{w}_{\mathrm{i}}$ & {$[\mathrm{cm}]$} & Length of a single volume element \\
\hline $\mathrm{V}$ & {$\left[\mathrm{g} / \mathrm{cm}^{3}\right]$} & Total vertebrae volume \\
\hline$\overline{W_{P A}}$ & {$\left[\mathrm{~g} / \mathrm{cm}^{2}\right]$} & The average vertebral width in PA view \\
\hline$W_{P A}^{\min }$ & {$[\mathrm{cm}]$} & The minimal vertebrae width in PA projection \\
\hline$\overline{W_{L A T}}$ & {$[\mathrm{~cm}]$} & Average LAT vertebral width \\
\hline aBMD & {$\left[\mathrm{g} / \mathrm{cm}^{2}\right]$} & $\begin{array}{l}\text { Areal bone mineral density of the vertebrae, } \\
\text { the result of standard DXA study }\end{array}$ \\
\hline vBMD & {$\left[\mathrm{g} / \mathrm{cm}^{3}\right]$} & $\begin{array}{l}\text { Volumetric bone mineral density of the } \\
\text { vertebrae, the result of QCT study }\end{array}$ \\
\hline $\mathrm{vBMD}_{\min }$ & {$\left[\mathrm{g} / \mathrm{cm}^{3}\right]$} & $\begin{array}{l}\text { Volumetric bone mineral density of the vertebrae } \\
\text { estimated with the use of the minimal PA } \\
\text { width }\end{array}$ \\
\hline $\mathrm{vBMD}_{\mathrm{av}}$ & {$\left[\mathrm{g} / \mathrm{cm}^{3}\right]$} & $\begin{array}{l}\text { Volumetric bone mineral density of the vertebrae } \\
\text { estimated with the use of the average PA width }\end{array}$ \\
\hline BMAD & {$\left[\mathrm{g} / \mathrm{cm}^{3}\right]$} & $\begin{array}{l}\text { Bone mineral apparent density estimated with the } \\
\text { use of A }\end{array}$ \\
\hline $\mathrm{K}$ & & Constant \\
\hline
\end{tabular}

vBMD describes the bone mineral content in the whole vertebrae volume and can be expressed as:

$$
v B M D=\frac{B M C}{V}=\frac{B M C}{a \cdot \sum_{i=1}^{N} w_{i}}=\frac{B M C}{a \cdot N \cdot \overline{W_{L A T}}}
$$

If we consider (1) and (2):

$v B M D=\frac{a B M D}{\overline{W_{L A T}}}$

The average LAT width cannot be measured in PA geometry most frequently used in vertebral DXA. It could be measured additionally in LAT view, but this requires another measurement, and our aim is to estimate the BS in a single PA DXA study. In order to avoid the LAT measurement, we assumed that despite the vertebrae size, all human vertebrae 


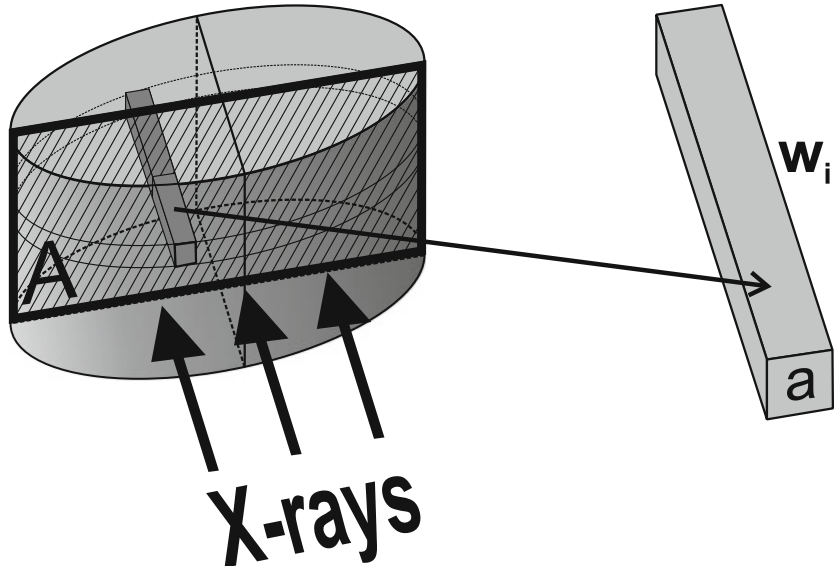

Fig. 1 Schematic view of DXA vertebral measurement geometry. The vertebra is scanned in the postero-anterior view. For mathematical description used in the present work, the whole vertebrae body was divided into $\mathrm{N}$ long and narrow pieces. The base area of each element is a, while their length is different for every element (denoted as $\mathrm{w}_{\mathrm{i}}$ ). The whole vertebrae body projection area is $\mathrm{A}$

are similar in shape, so the average PA and LAT widths are linearly dependent:

$\overline{W_{L A T}}=K \cdot \overline{W_{P A}}$

An increased vertebral AP diameter for older patients is a phenomenon not reported to the extent that could possibly make our assumption not valid, so the relation presented in Eq. (4) was checked by means of Pearson's correlation (see sections Results and Discussion).

Considering (3) and (4):

$K \cdot v B M D=\frac{a B M D}{\overline{W_{P A}}}$

$\mathrm{K}$ is a constant that is unknown. It would be easy to calculate $\mathrm{K}$ in geometry, as presented in Fig. 1, but not in the real anatomy. $\mathrm{K}$ considers the fact that $\mathrm{QCT}$ and DXA are in fact different methods and use different geometries. DXA measures the bone mineral content in the projected vertebrae area (containing the posterior vertebrae elements and considering the trabecular as well as the cortical bone), while QCT usually measures (but not always) the mineral content in the elliptical central part of the vertebrae containing the trabecular bone. Let's define a new parameter:

$v B M D_{a v}=\frac{a B M D}{\overline{W_{P A}}}$

Equation (6) describes an estimator of volumetric mineral density, which is linearly connected to $\mathrm{vBMD}\left(\mathrm{vBMD}_{\mathrm{av}}=\mathrm{K}\right.$. vBMD) and which could be calculated from DXA results. The usefulness of $\mathrm{vBMD}_{\mathrm{av}}$ as $\mathrm{BS}$ predictor will be investigated.

$\overline{W_{P A}}$ is measured as the additional parameter in the standard DXA procedure. In our studies $\overline{W_{P A}}$ was estimated as the average of the PA vertebrae widths measured every $1 \mathrm{~mm}$ along the entire vertebrae height. There is a remaining question-whether $\overline{W_{P A}}$ is the best geometrical parameter measured in PA view for $\overline{W_{L A T}}$ replacement. Additionally, we also considered the minimal PA width $\left({W_{P A}}^{\mathrm{min}}\right)$ as the PA estimator of $\overline{W_{L A T}}$, so finally, except from the index defined in (6), we also considered another one:

$v B M D_{\min }=\frac{a B M D}{W_{P A}^{\min }}$

Twenty-five cadaveric L3 vertebrae from males aged 22 81 years were studied. Studied material was collected in accordance with the guidelines of the proper ethics commission. All vertebrae were embedded in plastic containers (20 $\mathrm{cm}$ in diameter) filled with $0.9 \% \mathrm{NaCl}$ solution to simulate soft tissue and investigated with CT, QCT, and DXA. In order to minimize the potential influence of cadaver preservation on bone mechanical properties, all measurements were performed as soon as possible after acquirement (no more than 4 days).

A Siemens Somatom Sensation 10 (Siemens, Munich, Germany) CT unit was used for CT and QCT measurements (slice thickness, $0.6 \mathrm{~mm}, 120 \mathrm{kV}, 120 \mathrm{mAs}$ ). Images were reconstructed using an ultra-sharp reconstruction kernel. A $75 \times 75-\mathrm{mm}^{2}$ field of view and a matrix of $512 \times 512$ resulted in a pixel size of $146 \times 146 \mu \mathrm{m}^{2}$ in the plane of the scan. Volumetric bone mineral density was measured for cancellous region within vertebral body using Siemens Osteo-CT software. The trabecular tissue properties are widely believed to be the most relevant to FR $[1,6,15,16,19]$. The vBMD was measured by comparing the average $\mathrm{HU}$ of the chosen elliptical regions of interest within the vertebral bodies with similar areas within Siemens density standards.

The aBMD of each vertebra was measured with Lunar DPX-IQ (Lunar, Madison, US) following standard procedures applied for humans.

Geometrical measurements of the examined vertebrae were performed on the reconstructed three-dimensional CT images. DICOM data acquired in CT studies were imported and reconstructed by self-developed software based on the OpenGL library. Software developed in our laboratories allowed for fast, precise, and comfortable three-dimensional images manipulations (rotations, shifts) and measurements of linear, areal, and volumetric dimensions.

$\overline{W_{L A T}}$ and $\overline{W_{P A}}$ were estimated appropriately on sagittal and coronal CT cross-sections. Vertebrae widths were measured every $1 \mathrm{~mm}$ along the whole vertebrae heights and finally the average values were calculated. Additionally, the axial cross-sectional area $\left(A_{a}{ }^{\mathrm{min}}\right)$ in the most narrow vertebrae site was determined.

Mechanical vertebrae properties were tested by Instron 5566 testing device (Instron, High Wycombe, UK). In order 
to make the mechanical test possible and to ensure that our results will be comparable to other authors' results; likewise, sample preparation similar to that described elsewhere was applied [1, 6, 8, 11, 19-21]. The posterior vertebrae elements were removed to fix samples to the testing device and to ensure that loading will be distributed as in case of vertebral fractures in vivo mechanisms [21]. It is important to remember that the contribution of posterior elements to the vertebral strength has been evaluated in $0-57 \%$ [21]. In order to allow a uniform distribution of the stress on the bone specimen, two layers of acrylic resin were placed on the top and bottom endplates of the vertebrae $[1,6,8,11,19-21]$. After ten cycles of preloading, a constant deformation at the rate of $5 \mathrm{~mm} / \mathrm{min}$ was applied, and displacement-load curves were collected. The value of a load crashing the vertebrae, $\left(\mathrm{F}_{\max }\right)$ - ultimate load, was extracted from the displacement-load curves. The ultimate stress $\left(\mathrm{P}_{\max }\right)$ was also considered and calculated. It was assumed that vertebrae crashes in a plane where the maximal stress occurs, so the maximal stress was calculated as $[19,20]$ :

$P_{\max }=\frac{F_{\max }}{A_{A}^{\min }}$

Our study was aimed for the assessment of BS (and potentially FR) for patients who did not suffer from vertebrae fractures in the past and not for the fractures diagnosis. The mechanical vertebrae properties are definitely influenced by possible past fractures, so the geometrical measurements and a semi-quantitative method of the vertebrae fracture assessment $[22,23]$ were used to eliminate the vertebrae suspected for past fractures. Two specimens were excluded as suspected for the previous fractures. Three others were excluded because their displacement-load curves did not allow for the ultimate stress calculations. In these cases, the maximal mechanical testing device load $(20 \mathrm{kN})$ was achieved without vertebrae disruption. An example of such a case is denoted by an asterisk in Fig. 2. In total, 20 specimens were considered.

$\mathrm{F}_{\max }$ and $\mathrm{P}_{\max }$ as the estimators of $\mathrm{BS}$ were correlated with aBMD, vQCT, $\mathrm{vQCT}_{\text {min }}$, and $\mathrm{vQCT}_{\mathrm{av}}$ by Pearson's correlation. Because of a wide vertebrae donor's age range, the partial correlations were calculated in order to perform the age adjustment. Obtained correlation coefficients were compared, and the statistical significance of their differences was tested with using Hostelling's test.

\section{Results}

A few examples of load-displacement curves acquired for investigated samples are shown in Fig. 2. They were applied for the calculations of $F_{\max }$ and $P_{\max }$ subsequently.

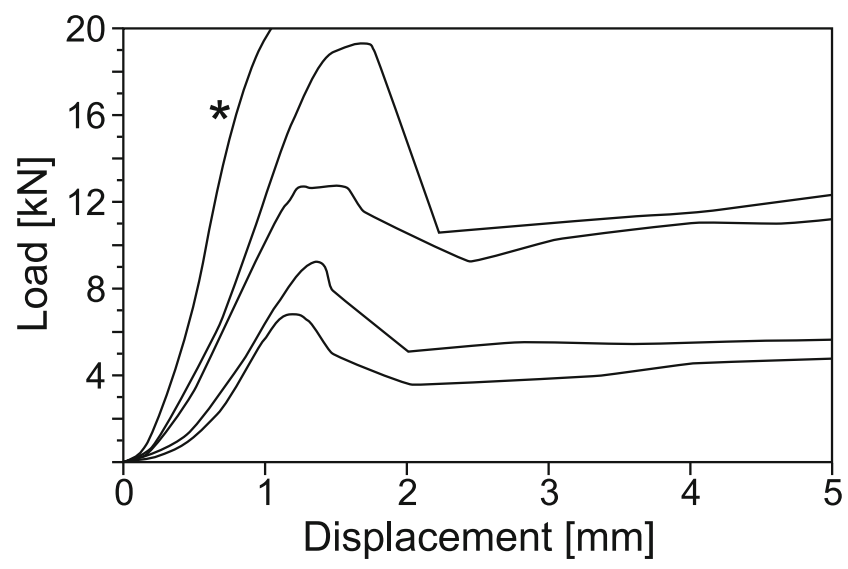

Fig. 2 Displacement-load curves acquired for four example samples. The curve marked by an asterisk represents the case when the maximal testing device load $(20 \mathrm{kN})$ was achieved and the vertebrae remained unbroken

The Pearson's correlation coefficients of $\mathrm{F}_{\max }$ and $\mathrm{P}_{\max }$ to all considered indexes describing bone densities are presented in Tables 2 and 3. Calculated correlation coefficients revealed statistically significant linear correlations to both $\mathrm{F}_{\max }$ and $\mathrm{P}_{\max }(p<0.05)$. The plots of estimated densities in function of $F_{\max }$ and $\mathrm{P}_{\max }$ are presented in Figs. 3 and 4, respectively.

As expected $\mathrm{vBMD}$ offered the strongest correlation to $\mathrm{P}_{\max }(r=0.883) . \mathrm{vBMD}_{\min }$ and $\mathrm{vBMD}_{\mathrm{av}}$ correlated slightly worse ( $r=0.842$ and $r=0.837$, respectively) than vBMD but considerably better than BMAD $(r=0.799)$ and aBMD $(r=0.779)$. BMAD seems to be a better BS predictor than aBMD. The Hostelling's tests showed that all correlation coefficient differences were statistically significant. The correlations to maximal load $\left(\mathrm{F}_{\max }\right)$ are worse in all cases compared to $\mathrm{P}_{\max }$ but are still statistically significant.

The partial correlation coefficients and $p$ values after the donor's age adjustment are shown in last two columns of Tables 2 and 3. After adjustment for age, the correlations remained statistically significant only for $\mathrm{P}_{\max }(p<0.05)$ while the statistical significance of correlation to $F_{\max }$ was lost. The adjusted correlation of $\mathrm{vBMD}$ and $\mathrm{P}_{\max }$ still remains the strongest $(r=0.648)$ and aBMD correlation is the weakest $(r=0.511)$. $\mathrm{vBMD}_{\min }$ correlates a slightly worse to $\mathrm{P}_{\max }$ than

Table 2 The results of statistical analysis of correlations between considered bone quality parameters and ultimate load $\left(\mathrm{F}_{\max }\right)$ (see text for parameters definitions)

\begin{tabular}{llrll}
\hline & $\begin{array}{l}\text { Pearson's } \\
\text { coefficient }\end{array}$ & $p$ value & $\begin{array}{l}\text { Partial correlation after } \\
\text { donors' age adjustment }\end{array}$ & $p$ value \\
\hline vBMD & 0.816 & $<0.0001$ & 0.500 & 0.03 \\
vBMD $_{\text {min }}$ & 0.729 & 0.0003 & 0.370 & 0.12 \\
vBMD $_{\text {av }}$ & 0.747 & 0.0002 & 0.408 & 0.09 \\
BMAD & 0.738 & 0.0002 & 0.439 & 0.06 \\
aBMD & 0.762 & 0.0001 & 0.490 & 0.04 \\
\hline
\end{tabular}


Table 3 The results of statistical analysis of correlations between considered bone quality parameters and ultimate stress $\left(\mathrm{P}_{\max }\right)$ (see text for parameters definitions)

\begin{tabular}{llcll}
\hline & $\begin{array}{l}\text { Pearson's } \\
\text { coefficient }\end{array}$ & $p$ value & $\begin{array}{l}\text { Partial correlation after } \\
\text { donors' age adjustment }\end{array}$ & $p$ value \\
\hline vBMD & 0.883 & $<0.0001$ & 0.648 & 0.003 \\
vBMD $_{\text {min }}$ & 0.842 & $<0.0001$ & 0.626 & 0.005 \\
vBMD $_{\text {av }}$ & 0.837 & $<0.0001$ & 0.610 & 0.006 \\
BMAD $_{\text {aBMD }}$ & 0.799 & $<0.0001$ & 0.567 & 0.011 \\
aBMD & 0.779 & 0.001 & 0.511 & 0.03 \\
\hline
\end{tabular}

vBMD, but better than $\mathrm{vBMD}_{\mathrm{av}}$ and significantly better than BMAD and aBMD.

In order to check if the replacement of $\overline{W_{L A T}}$ by $\overline{W_{P A}}$ and by $W_{P A}{ }^{\text {min }}$ we assumed was reasonable, the appropriate Pearson's correlation coefficients were calculated. $\overline{W_{P A}}$ and $\overline{W_{L A T}}$ correlate with the coefficient of $0.682(p=0.002)$ while $W_{P A}{ }^{\min }$ correlates to $\overline{W_{L A T}}$ with the strength of 0.705 $(p=0.0009)$.

In order to explain the differences in $\mathrm{BMAD}$ and $\mathrm{vBMD}_{\min }$ abilities for $\mathrm{P}_{\max }$ variability prediction, the correlation between $\overline{W_{L A T}}$ and A was also calculated and was equal to $0.673(p=0.0011)$.

\section{Discussion}

From a clinical point of view, the prediction of FR is one of the most important issues in bone diseases. Owing to the fact that FR is strongly dependent on the BS, we focused on it instead of the weakly defined FR. In the present study, we proved that there is a strong correlation between $\mathrm{BS}$ and volumetric bone mineral density estimated from PA DXA measurements. Two parameters estimating volumetric density from PA DXA were involved, and both of them correlated better to BS, represented by the ultimate stress, than aBMD. It was also confirmed that BMAD described elsewhere $[10,15,17,18]$ is a better predictor of $\mathrm{BS}$ than $\mathrm{aBMD}$.

The $\mathrm{vBMD}_{\mathrm{av}}$ calculated as the DXA aBMD normalized by the average PA vertebral body width is similar to the parameter described by Kroger et al. [24] and Jergas et al. [13] and used in other works $[15,16]$. The $\mathrm{vBMD}_{\mathrm{av}}$ correlated well with $\mathrm{P}_{\max }(r=0.610)$. The correlation of vBMD to $\mathrm{P}_{\max }$ measured
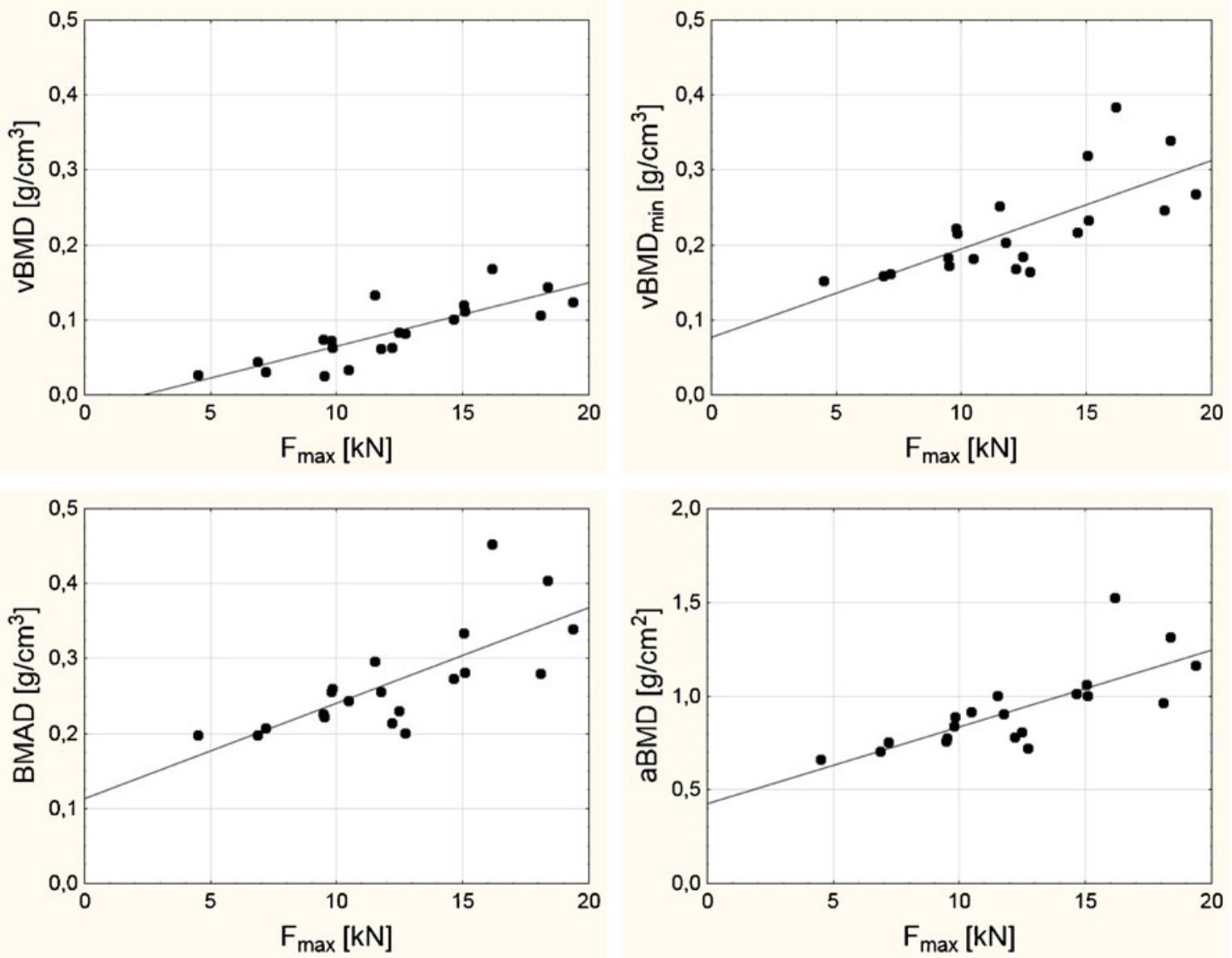

Fig. 3 vBMD, vBMD $D_{\min }$, BMAD, and aBMD plotted as a function of ultimate load $\left(\mathrm{F}_{\max }\right)$. The fitted linear functions are presented. For parameters definitions, see Table 1 . Correlation coefficients of presented dependencies are contained in Table 2 

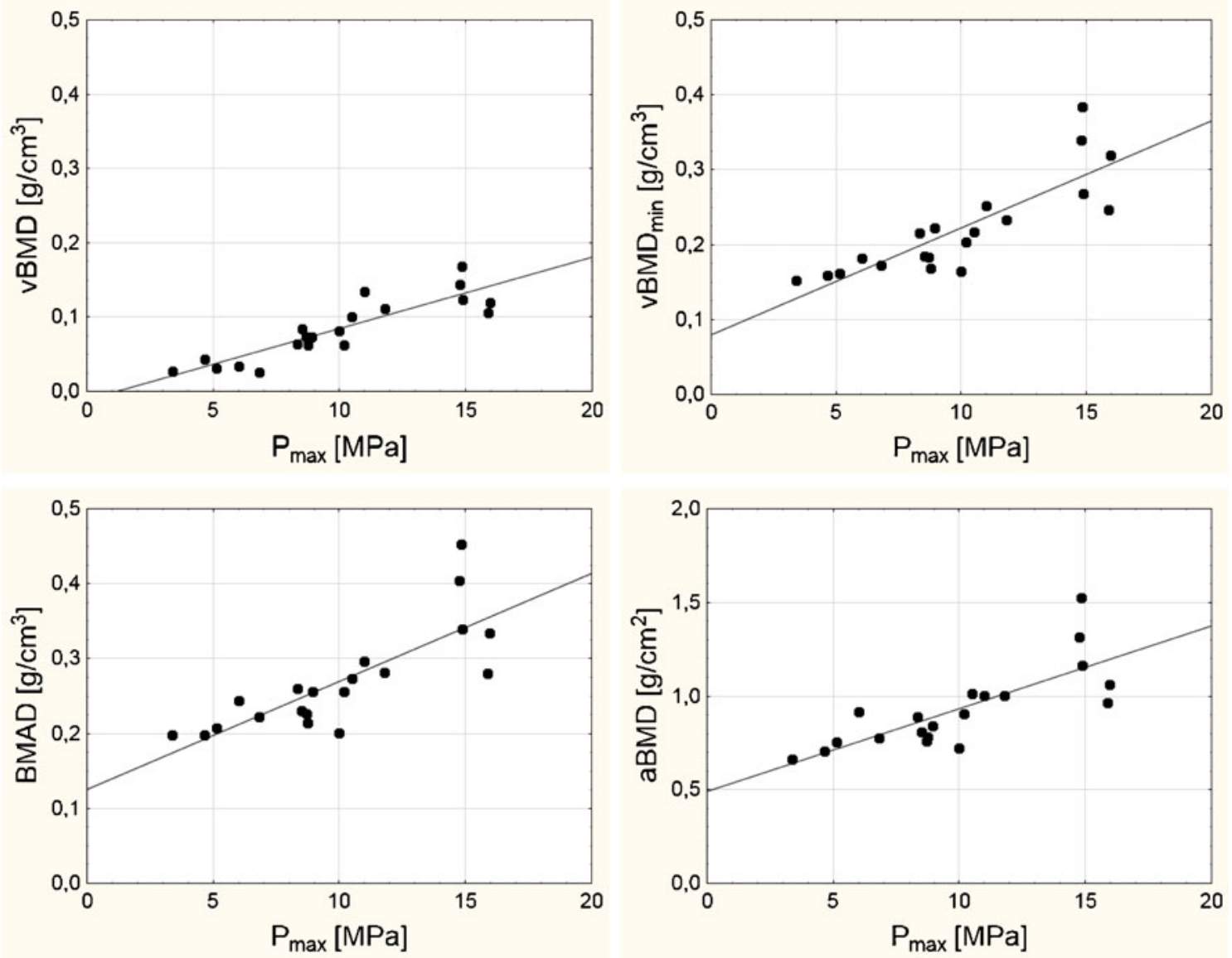

Fig. 4 vBMD, vBMD ${ }_{\min }$, BMAD, and aBMD plotted as a function of ultimate stress $\left(\mathrm{P}_{\max }\right)$. The fitted linear functions are presented. For parameters definitions, see Table 1. Correlation coefficients of presented dependencies are contained in Table 3

directly by $\mathrm{QCT}$ is higher than in case of $\mathrm{vBMD}_{\mathrm{av}}$ and is equal to 0.648 . On the other hand, $\mathrm{vBMD}_{\mathrm{av}}$ is considerably more accurate than aBMD $(r=0.511)$ in BS variability prediction. All of these findings agree with previously published data [13, $15,16,24]$.

We proposed a modification of aBMD geometrical correction. Instead of the average vertebral body width, we proposed the mid-vertebral body width for DXA aBMD normalization. The parameter achieved in this way $\left(\mathrm{vBMD}_{\min }\right)$ has stronger correlation to $\mathrm{P}_{\max }$ than $\mathrm{vBMD}_{\mathrm{av}}$. The $\mathrm{vBMD}_{\min }$ describes BS variability better than $\mathrm{vBMD}_{\mathrm{av}}$ probably due to the fact that the former contains the information about the mid-vertebral region, which is cross-sectionally characterized by the narrowest axial area distributing the highest stress, so it is especially susceptible to fractures [19].

Both $\mathrm{vBMD}_{\min }$ and $\mathrm{vBMD}_{\mathrm{av}}$ correlate positively to $\mathrm{BS}$, which means that the higher values of these parameters translate into higher BS and lower FR.

The aBMD adjusted by the vertebral body size improved the BS prediction and probably also FR prediction. Many authors conclude that aBMD cannot be the single parameter influencing BS, but bone dimensions, shape, structure, and an individual patient's history should also be considered in the assessment of FR [1, 2, 4-6, 8, 16, 19, 23, 25]. The idea of taking into consideration geometrical parameters in order to support aBMD acquired in DXA and to improve its diagnostic value has been already examined. The combination of aBMD and other parameters, including geometrical, were considered for different skeletal sites in FR prediction. Yu et al. [26] showed in an in vivo study that femoral aBMD, femoral neck width, and body weight could be combined to explain variability in the risk of hip fractures. Some authors claim that it is not enough to measure only aBMD or bone mineral content (BMC) of the forearm to predict the BS of the distal radius. A clinical assessment of BMC and/or cross-sectional area in the radius would provide improved prediction of an individual risk of radius fracture [27].

There are also some data on the vertebral FR assessment utilizing other parameters in addition to aBMD. Most of them support our conclusions $[2,10,15,19,28]$, but not all. Cheng et al. [19] showed that a combination of aBMD measured in DXA with vertebral body cross-sectional area considerably increases the ultimate load variability prediction. This is contrary to our findings because we did not observe a statistically 
significant correlation between considered volumetric estimators to $\mathrm{F}_{\max }$. Even the correlation of vBMD measured in means of QCT was on the significance border $(r=0.500)$. On the other hand, Cheng et al. also showed that vBMD predicts the vertebral ultimate stress more efficiently than aBMD, which is in agreement with our data.

Ross et al. [28] showed in vivo studies that a combination of large vertebral depth and low bone density increases considerably the vertebrae FR. Their results support our findings. The larger vertebral depth (estimated here by $\overline{W_{P A}}$ ) accompanied by lower aBMD translate into lower $\mathrm{vBMD}_{\min }$ and $\mathrm{vBMD}_{\mathrm{av}}$. Both indices correlate positively with the vertebral $\mathrm{BS}$, so lower volumetric densities also mean a lower BS, and a higher FR.

Wren et al. [10] described two aBMD geometrical adjustments aiming at the $\mathrm{vBMD}$ and $\mathrm{BMC}$ assessment from PA DXA. They defined $v B M D 1=a B M D / \sqrt{A}$ and $v B M D 2=$ $\mathrm{aBMD} / \mathrm{H}$ ( $\mathrm{H}$ is the vertebral body height). vBMD1 is in fact the same as BMAD considered here and described by other authors $[15,17,18]$. Wren and his group found high correlations between BMC based on DXA adjusted by geometry and QCT but association between vBMD estimated from QCT and aBMD obtained from DXA was insufficient. Calculation of vBMD1 and vBMD2 only slightly improved the correlation to QCT vBMD. The parameters we describe improve the correlation to BS considerably, but it is difficult to compare these two works as Wren et al. correlated their parameters to vBMD and not to $\mathrm{P}_{\max }$ as we did. Both vBMD and $\mathrm{P}_{\max }$ roughly describe BS, but they represent different physical values.

Dowthwaite and his group [16] compared a group of exgymnastic and non-gymnastic women in means of vertebral geometry and vertebral bone density. They stated that the gymnastic group was characterized by shorter but wider vertebral bodies as well as higher aBMD and BMC in comparison to the non-gymnastic group. The calculated FRAX was smaller for gymnastic group. These results are a little bit confusing for our conclusions because we normalized the aBMD by $\overline{W_{P A}}$ as the estimator of $\overline{W_{L A T}}$. We assumed the positive correlation between the last two, so according to our findings, the smaller values of $\overline{W_{P A}}$ (and $\overline{W_{L A T}}$ ) mean higher BS. According to Dowthwaite, $\overline{W_{P A}}$ increment increases BS while $\overline{W_{L A T}}$ increment decreases BS [16].

There is a problem connected to the assumption of a correlation between $\overline{W_{P A}}$ and $\overline{W_{L A T}}$. It is well known that the vertebral dimensions change with a patient's age, e.g., Briot et al. studied and observed the vertebral LAT dimension changes [4]. Older vertebrae seem to be wider than younger, but there is an open question about whether the ratio of LAT and AP widths also changes with a patient's age. To the best of our knowledge, there are no reports suggesting larger increment in AP vertebral dimensions than in LAT dimensions. We checked $\overline{W_{P A}}$ and $W_{P A}{ }^{\text {min }}$ to $\overline{W_{L A T}}$ correlations and obtained quite high coefficients, but in order to avoid any doubt, we must conclude that this requires further study. The best possibility would be not $\overline{W_{L A T}}$ estimation but additional DXA measurement in LAT view [15, 16], allowing direct $\overline{W_{L A T}}$ measurement. Leonard et al. [15] estimated the vBMD in children pairing the PA and LAT DXA results. They applied LAT and PA vertebral sizes in order to calculate the vertebrae volume and vBMD. They concluded that paired PA and LAT scans may increase sensitivity to real trabecular BMC and density variability.

Dall'Ara et al. [8] recently performed in vitro studies concerning the prediction of vertebral BS by finite elements modeling (FEM). They compared the ability of FEM on this field to vBMD achieved from QCT and aBMD. The PA and LAT aBMD was calculated from high-resolution, threedimensional images in a way that they would be measured in the real DXA study. They concluded that FEM has the greatest ability to properly predict vertebrae mechanical properties, but their findings concerning vBMD and aBMD are interesting in our study context. Accordingly to the Dall'Ara group [8], aBMD in both PA and LAT views are better associated with $\mathrm{F}_{\max }$ than vBMD. There is an opposite relationship when considering $\mathrm{P}_{\max }$, vBMD much better predicts ultimate stress than both aBMDs. Their findings were partially confirmed in our results. The correlations between vBMD (and also three described vBMD estimators) are higher than in case of aBMD if consider $\mathrm{P}_{\max }$. Contrary to Dall'Ara's results, the $\mathrm{vBMD}$ of our samples was the best predictor also for $F_{\text {max }}$, but aBMD acts better than $\mathrm{vBMD}_{\min }, \mathrm{vBMD}_{\mathrm{av}}$, and BMAD. The discrepancy between the results of that study [8] and our results was probably caused by different approaches to vBMD estimation. They used total volumetric bone mineral density, taking into account trabecular as well as cortical bone, while we considered in our studies only the cancellous part. This conclusion is speculative and needs further investigation, but if our results would be confirmed, it would mean that vBMD estimated for trabecular bone only is a better choice than the measurement of both trabecular and cortical densities.

Beside $\mathrm{vBMD}_{\text {min }}$ and $\mathrm{vBMD}_{\mathrm{av}}$ proposed here, BMAD proposed by other authors $[10,15,17,18]$ was also investigated. aBMD variability is caused not only by the real BMC changes but also by body size variability [17]. That problem is especially important for children and adolescents because during growth, fast BMC changes are accompanied by fast changes in stature $[10,18]$. BMAD was introduced as a potential solution for this problem. Our results showed that aBMD corrected by $A$ is better associated to $P_{\max }$ than aBMD alone, but $\mathrm{vBMD}_{\min }$ and $\mathrm{vBMD}_{\mathrm{av}}$ explain $\mathrm{P}_{\max }$ variability more accurate. If we consider the ultimate load prediction, BMAD fails as $\mathrm{vBMD}_{\min }$ and $\mathrm{vBMD}_{\mathrm{av}}$ failed compared to aBMD and vBMD. Vertebrae fracture is the effect dependent not only on external loads but also on the vertebrae properties such as BMC and dimensions. Therefore, we believe the 
prediction of $\mathrm{P}_{\max }$ variability has a higher importance than $F_{\max }$ variability prediction and such parameters such as BMAD, $v B M D_{\text {min }}$, and $\mathrm{vBMD}_{\mathrm{av}}$ are still worth considering. Our results showed that $\mathrm{vBMD}_{\min }$ explains $\mathrm{P}_{\max }$ variability much better than BMAD does. This is probably connected to the fact that $W_{P A}{ }^{\min }$ (used for $\mathrm{vBMD}_{\text {min }}$ assessment) reflects the vertebrae geometry changes in LAT view more accurate than A used for BMAD calculations. It is visible if we compare correlations of $\overline{W_{L A T}}$ to $\mathrm{A}$ $(r=0.673)$ and to $W_{P A}{ }^{\min }(r=0.705)$.

The main limitation of the present study is the low number of vertebrae studied. More reliable conclusions could be derived from a larger population sample, especially involving wider age ranges. It should also be clearly pointed out that our results were obtained in vitro and conclusions for in vivo BS or FR assessment are rather speculative. The potential clinical application of our conclusions requires additional in vivo studies. Because it is impossible to measure ultimate stress or load in vivo, such investigations should consider the dependencies between proposed indices and FRAX.

In this in vitro study, we confirmed that a combination of aBMD measured in means of DXA with geometrical vertebrae measurement allows for more accurate vertebrae mechanical strength prediction than if only DXA results are analyzed. The aBMD normalized by the vertebrae width correlates better to BS than aBMD alone.

The novel parameter proposed in our study utilizes the mid-vertebral body width for aBMD normalization and performs better in BS prediction than if the average vertebral body width or the PA bone area would be applied.

Applying single DXA scanning one can achieve improved description of vertebral BS comparing to aBMD. Better performance probably would be achieved if both PA and LAT scans could be analyzed.

Conflict of interest The authors declare that they have no conflicts of interest.

Open Access This article is distributed under the terms of the Creative Commons Attribution License which permits any use, distribution, and reproduction in any medium, provided the original author(s) and the source are credited.

\section{References}

1. Ammann P, Rizzoli R. Bone strength and its determinants. Osteoporos Int. 2003;14 Suppl 3:S13-8.

2. Diacinti D, Pisani D, Barone-Adesi F, et al. A new predictive index for vertebral fractures: the sum of the anterior vertebral body heights. Bone. 2010;46:768-73.

3. Pulkkinen P, Partanen J, Jalovaara P, Jamsa T. Combination of bone mineral density and upper femur geometry improves the prediction of hip fracture. Osteoporos Int. 2004;15:274-80.
4. Briot K, Kolta S, Fechtenbaum J, Said-Nahal R, Benhamou CL, Roux C. Increase in vertebral body size in postmenopausal women with osteoporosis. Bone. 2010;47:229-34.

5. Ruyssen-Witrand A, Gossec L, Kolta S, Dougados M, Roux C. Vertebral dimensions as risk factor of vertebral fracture in osteoporotic patients: a systematic literature review. Osteoporos Int. 2007;18: 1271-8.

6. Crawford RP, Cann CE, Keaveny TM. Finite element models predict in vitro vertebral body compressive strength better than quantitative computed tomography. Bone. 2003;33:744-50.

7. Edwards WB, Troy KL. Number crunching: how and when will numerical models be used in the clinical setting? Curr Osteoporos Rep. 2011;9:1-3.

8. Dall'Ara E, Pahr D, Varga P, Kainberger F, Zysset P. QCT-based finite element models predict human vertebral strength in vitro significantly better than simulated DEXA. Osteoporos Int. 2012;23:563-72.

9. McCloskey E, Johansson H, Oden A, Kanis JA. Fracture risk assessment. Clin Biochem. 2012;45:887-93.

10. Wren TA, Liu X, Pitukcheewanont P, Gilsanz V. Bone acquisition in healthy children and adolescents: comparisons of dual-energy $\mathrm{x}$-ray absorptiometry and computed tomography measures. J Clin Endocrinol Metab. 2005;90:1925-8.

11. Lochmuller EM, Burklein D, Kuhn V, et al. Mechanical strength of the thoracolumbar spine in the elderly: prediction from in situ dualenergy X-ray absorptiometry, quantitative computed tomography (QCT), upper and lower limb peripheral QCT, and quantitative ultrasound. Bone. 2002;31:77-84.

12. Cheng XG, Lowet G, Boonen S, Nicholson PH, Van der Perre G, Dequeker J. Prediction of vertebral and femoral strength in vitro by bone mineral density measured at different skeletal sites. J Bone Miner Res. 1998;13:1439-43.

13. Jergas M, Breitenseher M, Gluer CC, Yu W, Genant HK. Estimates of volumetric bone density from projectional measurements improve the discriminatory capability of dual X-ray absorptiometry. J Bone Miner Res. 1995; 10:1101-10.

14. Carter DR, Bouxsein ML, Marcus R. New approaches for interpreting projected bone densitometry data. J Bone Miner Res. 1992; 7:137-45.

15. Leonard MB, Shults J, Zemel BS. DXA estimates of vertebral volumetric bone mineral density in children: potential advantages of paired posteroanterior and lateral scans. J Clin Densitom. 2006;9: 265-73.

16. Dowthwaite JN, Rosenbaum PF, Scerpella TA. Mechanical loading during growth is associated with plane-specific differences in vertebral geometry: a cross-sectional analysis comparing artistic gymnasts vs. non-gymnasts. Bone. 2011;49:1046-54.

17. Cvijetic S, Korsic M. Apparent bone mineral density estimated from DXA in healthy men and women. Osteoporos Int. 2004;15:295-300.

18. Zemel BS, Leonard MB, Kelly A, et al. Height adjustment in assessing dual-energy X-ray absorptiometry measurements of bone mass and density in children. J Clin Endocrinol Metab. 2010;95:1265-73.

19. Cheng XG, Nicholson $\mathrm{PH}$, Boonen $\mathrm{S}$, et al. Prediction of vertebral strength in vitro by spinal bone densitometry and calcaneal ultrasound. J Bone Miner Res. 1997;12:1721-8.

20. Singer K, Edmondston S, Day R, Breidahl P, Price R. Prediction of thoracic and lumbar vertebral body compressive strength: correlations with bone mineral density and vertebral region. Bone. 1995;17: 167-74.

21. Renau A, Farrerons J, Yoldi B, et al. Yield point in prediction of compressive behavior of lumbar vertebral body by dual-energy X-ray absorptiometry. J Clin Densitom. 2004;7:382-9.

22. Genant HK, Wu CY, van Kuijk C, Nevitt MC. Vertebral fracture assessment using a semiquantitative technique. J Bone Miner Res. 1993;8:1137-48.

23. Lewiecki EM. Bone densitometry and vertebral fracture assessment. Curr Osteoporos Rep. 2010;8:123-30. 
24. Kroger H, Vainio P, Nieminen J, Kotaniemi A. Comparison of different models for interpreting bone mineral density measurements using DXA and MRI technology. Bone. 1995;17: 157-9.

25. Edmondston SJ, Singer KP, Day RE, Price RI, Breidahl PD. Ex vivo estimation of thoracolumbar vertebral body compressive strength: the relative contributions of bone densitometry and vertebral morphometry. Osteoporos Int. 1997;7:142-8.
26. Yu N, Liu YJ, Pei Y, et al. Evaluation of compressive strength index of the femoral neck in Caucasians and Chinese. Calcif Tissue Int. 2010;87:324-32.

27. Parkinson IH, Fazzalari NL. Whole bone geometry and bone quality in distal forearm fracture. J Orthop Trauma. 2008;22:S59-65.

28. Ross PD, Huang C, Davis JW, Wasnich RD. Vertebral dimension measurements improve prediction of vertebral fracture incidence. Bone. 1995; 16:257S-62S. 